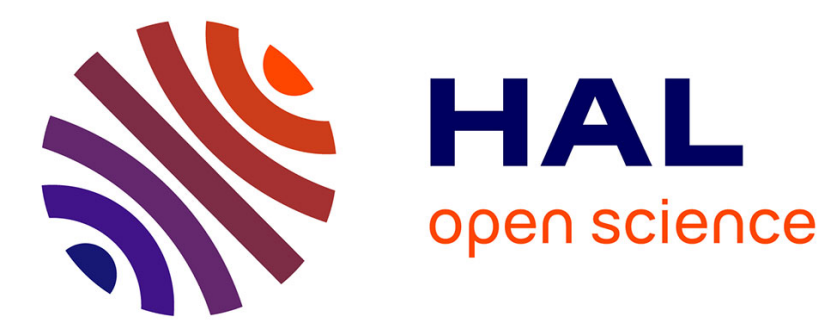

\title{
Pressure waves induced in liquid under high laser irradiation
}

\author{
A. Alloncle, D. Dufresne, M. Autric
}

\section{To cite this version:}

A. Alloncle, D. Dufresne, M. Autric. Pressure waves induced in liquid under high laser irradiation. Journal de Physique IV Proceedings, 1994, 04 (C4), pp.C4-131-C4-134. 10.1051/jp4:1994428 . jpa00252691

\section{HAL Id: jpa-00252691 https://hal.science/jpa-00252691}

Submitted on 1 Jan 1994

HAL is a multi-disciplinary open access archive for the deposit and dissemination of scientific research documents, whether they are published or not. The documents may come from teaching and research institutions in France or abroad, or from public or private research centers.
L'archive ouverte pluridisciplinaire HAL, est destinée au dépôt et à la diffusion de documents scientifiques de niveau recherche, publiés ou non, émanant des établissements d'enseignement et de recherche français ou étrangers, des laboratoires publics ou privés. 


\title{
Pressure waves induced in liquid under high laser irradiation
}

\author{
A.P. ALLONCLE, D. DUFRESNE and M. AUTRIC \\ Institut de Mécanique des Fluides, Equipe Interaction Laser-Matière, Parc Scientifique et Technologique \\ de Luminy, Avenue de Luminy, 13009 Marseille, France
}

\section{General topic}

It exists many mechanisms leading to soundwaves generation in liquid under a pulsed laser beam irradiation. The type of interaction, the soundwave amplitude and geometry depends on laser intensity, transparency of the medium to the incident radiation and geometry of the irradiated zone. Interaction occurs at the surface or inside the liquid, depending on its low or high absorption coefficient.

For low laser intensity, the energy absorption occurs without phase change. In this case, the mechanism inducing the highest pressure is thermoelasticity. It results from the thermic dilatation of a volume of liquid rapidly heated. In this case the pressure amplitude change linearly with energy.

Beyond a certain threshold, the breakdown occurs, associated or not with a previous vaporization phase. Linear variation does not exist any more between energy and amplitude of pressure wave.

This paper is concerned with an experimental study of the shock wave generated during the breakdown of a transparent liquid. This dielectric breakdown is characterized by an ionisation of the medium leading to the formation of a luminous plasma, which absorbs the incident radiation. Consequently, a shock wave is emitted and a small volume of liquid is heated and vaporized. This cavity, of initially high pressure and temperature, is following a cycle of expansion and collapse. It looses an important part of its energy between two cycles, which is dissipated in the medium as an acoustic wave. In good conditions of beam focusing and of liquid purity, the bubble becomes spherical very early in its expansion phase.

This method is very suitable for studying basic mechanisms of cavitation especially the nonspherical motion of bubbles in the vicinity of a solid boundary or near other cavitating bubbles [1][2][3][4][5].

Moreover, these type of research presents an increasing interest in the medical fields of ophthalmology and lithotripsy, where the same mechanisms are encountered.

This paper contributes to the different studies on laser generated vapour bubbles and shock waves, and it is particularly concerned with direct pressure measurements combining high speed visualisation and an interferometry. The main results describe the initial conditions of the bubble formation and the shock wave generation.

\section{Means and measures}

A schematic diagram of the experimental set-up is presented in Figure 1. Experiments are performed using a Nd:YAG laser, fitted with a frequency doubler, and delivering a pulse of 1,3J energy lasting 10ns (FHMW). The second harmonic is isolated using two dichroïc mirrors (M1 and M2). The beam is enlarged by a telescopic (T) system made of two confocal lenses. It is then focused into a cylindrical vessel of $20 \mathrm{~cm}$ diameter filled with demineralized water. The size of the spot at the focus is $36 \mu \mathrm{m}$. The breakdown occurs for an incident energy of $1 \mathrm{~mJ}$.

Visualisation was carried out using shadowgraph or on an interferometry with a a high-speed camera (Imacon 790 - Hadland) (framing rate up to $2.10^{7}$ frame/sec and streak speed up to $1 \mathrm{~ns} / \mathrm{mm}$ ). A continuous wave argon laser is used as an intense and monochromatic light source. The beam is divided into two equal parts. One of them is directed through the water, and records the disturbances of the medium induced by the breakdown, the pressure waves and the bubbles. The other one is used as a reference. Both beams are then directed to the photo cathode of the camera using a beam splitter. Accurate adjustments of the mirrors and the beam splitters cause interferences to occur.The fringe pattern is then recorded by the camera working at a framing rate of $2.10^{7}$ and $2.10^{6} \mathrm{frames} / \mathrm{sec}$ in which the exposure time is short enough to have a good resulting image. Every perturbation occurring in the visualized 


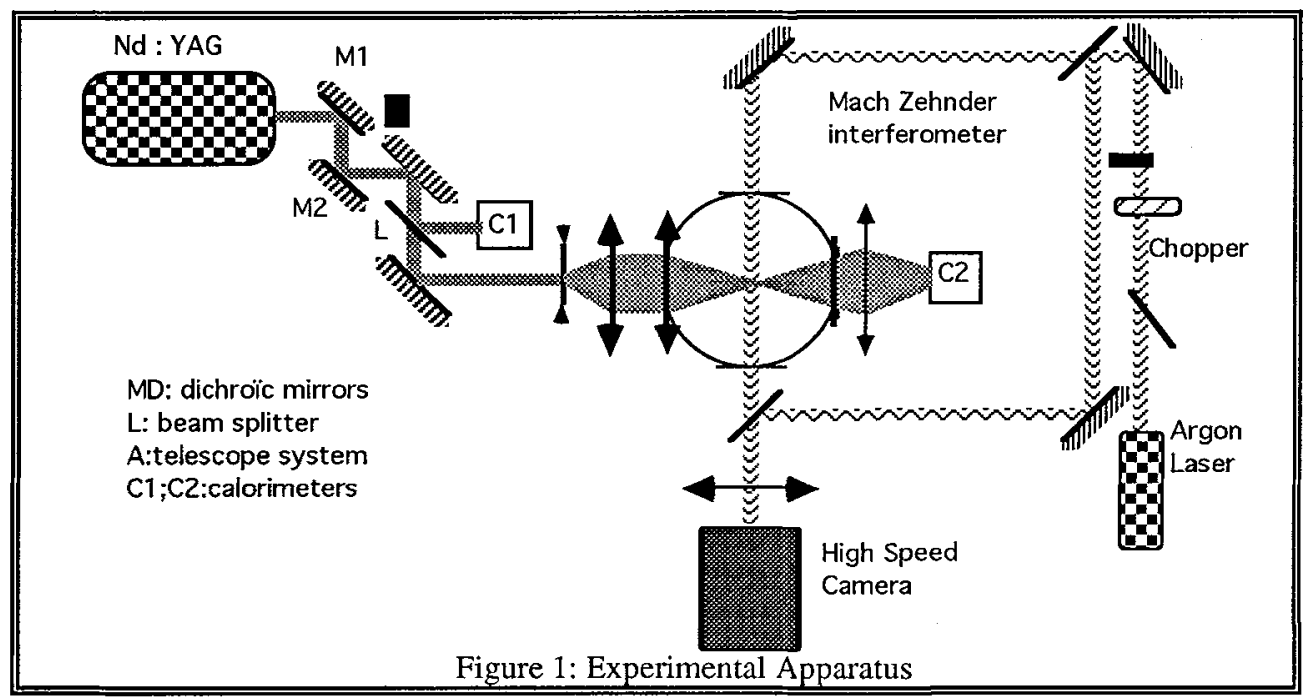

area leads to a fringe shift. A similar technique was used by Ward et al.[6] in optical cavitation experiments and gives interesting results.

The co-ordination of such an apparatus needs an accurate triggering device between the modulation of the continuous laser (performed using a shutter and a chopper), the pulsed laser and the camera.

Direct pressure measurements are taken from the interference pattern. Using an optical method instead of pressure gauges allows pressure measurements close to the centre of emission ( $200 \mu \mathrm{m}$ far from the centre of the interaction) to be obtained.

The pressure wave induces some variations of the refractive index along its propagation, consequently a shift of the fringe from the initial parallel pattern is recorded. The whole phenomenon demonstrates a spherical symmetry, and, it is thus possible to deduce the radial pressure profile from the fringe shift[7].

Pressure measurements using PVDF probes placed at $10 \mathrm{~mm}$ from the centre of the interaction were also taken in order to compare both techniques. PVDF is used because of very short rise times associated with the small size of the sensitive element $\left(1 \mathrm{~mm}^{2}\right)$.

The first nanoseconds of the phenomenon resulting from the laser/water interaction are visualisated in the streak mode with a fast recording speed $(1 \mathrm{~ns} / \mathrm{mm})$. The slit is pointed along the optical axis of the focusing system. Direct evolution with time of the breakdown, the shock wave and bubble interface motions are obtained.

\section{3 . Experimental results on initial conditions of laser generated bubble and shock wave}

In the focal zone the optical breakdown threshold of the medium is reached when the radiation intensity is maximum. Then, as the power pulse increases with time, the location of the breakdown moves to larger surfaces of the caustic, towards the incident beam according the "moving breakdown "model described by Docchio[8]. The ionization of the irradiated volume is associated with a recombination of the different elements inducing an emission of light. This phase is visualizated on fig $2 \mathrm{a}$ where the shielding effect of the plasma is very well shown. For our experimental conditions, very close to the breakdown threshold of the medium, the deposit of energy takes place with in a region of conical geometry of $130 \mu \mathrm{m}$ inlength. The duration of plasma luminescence is $6 \mathrm{~ns}$. The amount of deposited energy in the liquid varies over a very large range depending on the incident energy. From $1 \mathrm{~mJ}$ up to $4 \mathrm{~mJ}$ of incident energy, the ratio of deposited energy increases almost linearly up to $60 \%$. According to these results, an energy density in the range $10^{8}$ to $10^{9} \mathrm{~J} / \mathrm{m}^{3}$ can be supposed to create the shock wave and the bubble.

Consequent to this non spherical deposit of energy, an asymmetry appears in the development of the shock wave and the bubble as is illustrated on fig $2 \mathrm{~b}$. On this streak visualization 


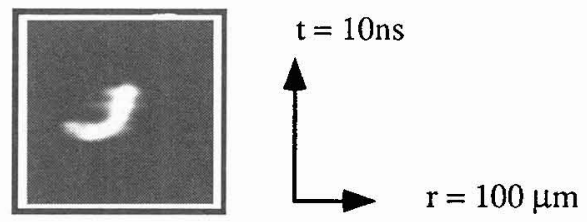

fig 2a: Streak visualization of the breakdown of the liquid. (laser beam comes from the right side)

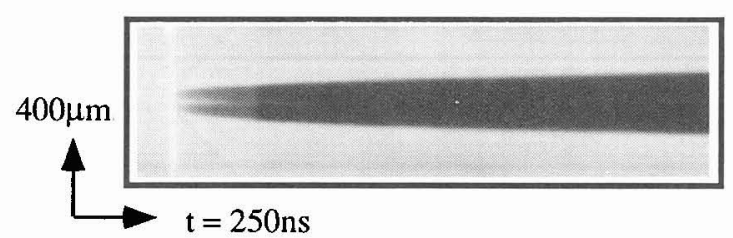

fig2b

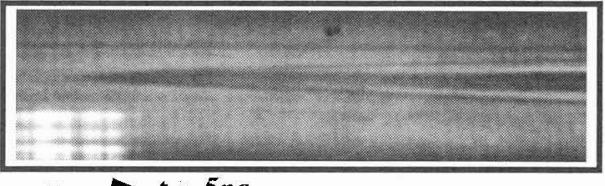

$\mathrm{t}=5 \mathrm{~ns}$

$$
\text { fig2c }
$$

fig $2 b$ and $2 c$ : streak visualization of the intial phase of the shock wave and bubble dynamics. (the laser beam comes from the top of both recordings)

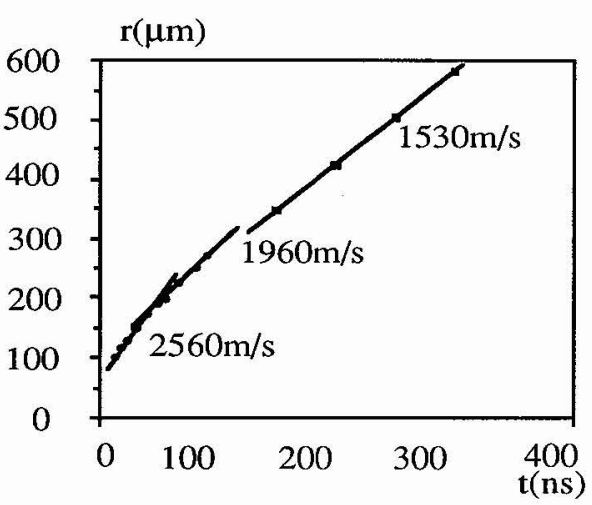

fig 2d:Shock wave velocity

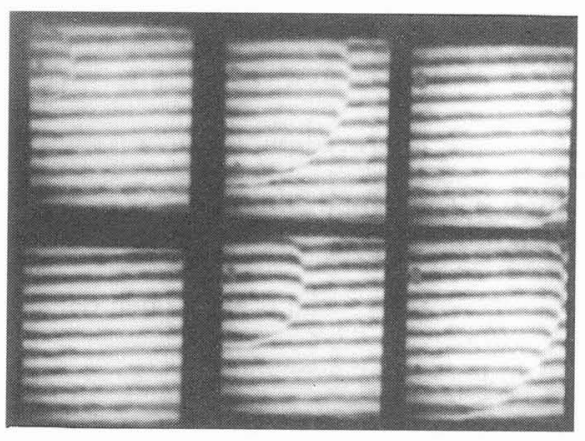

$$
\Delta \mathrm{t}=500 \mathrm{~ns}
$$

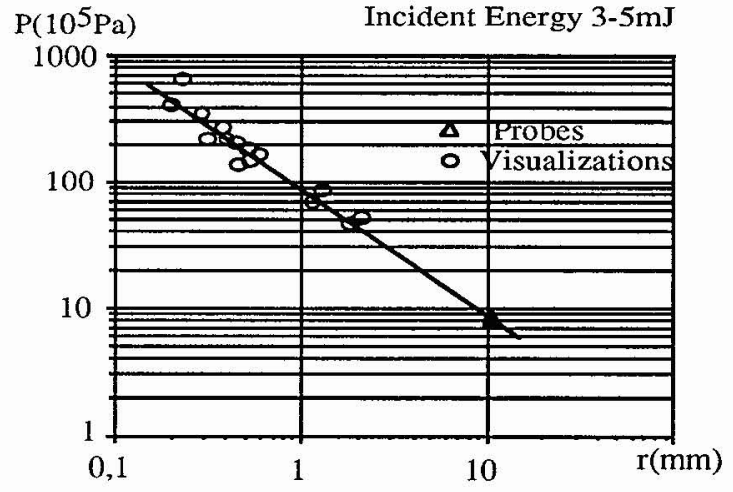

fig 3 : Variation of the pressure amplitude of as a function of the shock wave propagation

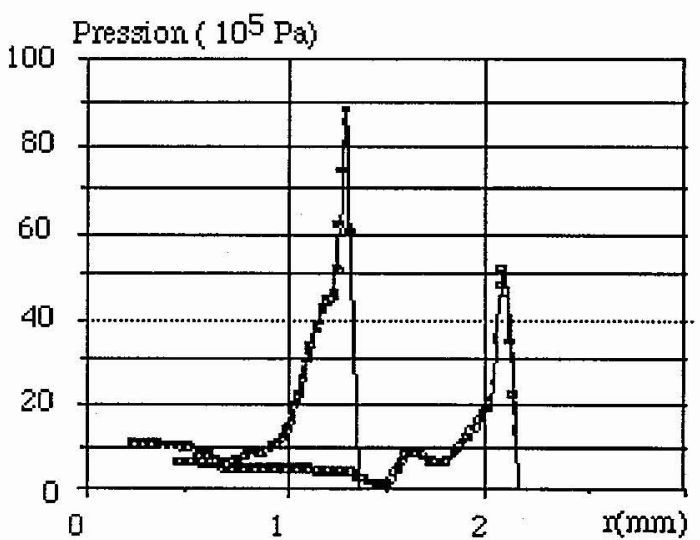

fig 4: Visualization of the spherical shock wave and pressure profile associated 
(1ns/mm), taken using a filter in order to hide the light of the plasma, the front shock can be distinguished from the bubble interface 10 to $20 \mathrm{~ns}$ after the beginning of the breakdown. The accuracy of the measurements depends here on the recording spacial resolution. The location of the shock wave as a function of time, is deduced from the visualization of fig $2 \mathrm{~b}$ and $2 \mathrm{c}$ and plotted on fig $2 \mathrm{~d}$. The initial velocity changes from $2100 \mathrm{~m} / \mathrm{s}$ to $2600 \mathrm{~m} / \mathrm{s}$ for the side directed toward the incident beam. Using the Hugonniot relations for water, an initial pressure of 7 to $1410^{8} \mathrm{~Pa}$ is calculated. The velocity decreases quickly in the medium to reach the sound velocity after $100 \mathrm{~ns}$. We can also consider that this is the characteristic time from when the geometry becomes spherical, and consequently the pressure amplitude decreases as a function of $1 / r$.

The pressure values plotted in fig 3 are taken from interferometric recordings and PVDF measurements. The dependence of the results on $1 / \mathrm{r}$ for both methods shows the validy of the optical process for pressure measurements. Interferometric measurements could be performed up to $200 \mu \mathrm{m}$ from the centre and pressures up to $50010^{5} \mathrm{~Pa}$ were recorded.

Figure 4 shows, as an example, visualizations (framing rate $500 \mathrm{~ns}$ ) and pressure profiles associated with $3-5 \mathrm{~mJ}$ of incident energy. The small grey point at the left upper corner of each pictures is the bubble and the shock wave is visualized by the fringe shift. The rises front is very short ( $60 \mathrm{~ns})$. The measurements are performed at $2.1 \mathrm{~mm}$ and $1.3 \mathrm{~mm}$ from the centre of the cavity, and the maximum pressure amplitudes are $50.105 \mathrm{~Pa}$ and $90.105 \mathrm{~Pa}$ respectively. The pressure then decreases slowly with a time constant of $70-90 \mathrm{~ns}$, to a value different from the undisturb hydrostatic pressure. The liquid is compressed between the shock wave and the bubble interface for a few microseconds, because of the explosive expansion of the bubble. The bubble's initial conditions of development are an interface velocity of $800 \mathrm{~m} / \mathrm{s}$ for a mean radius of $60 \mu \mathrm{m}$.

\section{Conclusion}

Interesting results have been taken using both high speed visualisation and interferometric pressure profile measurements, on the initial conditions of shock wave pressure and the bubble formation. These inital conditions are difficult to quantify for possible numerical simulations. The next step of our study is to compare our experimental results with a theoretical analysis of the vapour bubble and shock wave dynamics induced by laser deposit of energy.

These optical techniques have also been used for studying the collapse of a bubble. Interesting results have been obtained especially on the radial pressure profil of the acoustic transient due to the collapse[7]. It presents a symmetrical profile compared to the first shock wave, and the maximum of pressure amplitude in the wave of is less high.

\section{References}

1. W.Lauterborn, Cavitation and Inhomogenities in Underwater acoustics, Springer series in electrophysics 4, Springer-Verlag Berlin, Heidelberg, New-York 1980 pp3-12

2. P.Testud-Giovanneschi, D.Dufresne, Experimental study of laser induced cavitation bubbles, JAP vol 58 (2) pp651-652, July 1985

3. P.Testud-Giovanneschi, A.P.Alloncle, D.Dufresne, Collective effects of cavitation, Experimental study of bubble-bubble and bubble-shock wave interaction, J.Appl.Phys., vol $67, \mathrm{~N}^{\circ} 8,15$ April $1990, \mathrm{pp} 3560-3564$

4. Y.Tomita, A.Shima, H. Takahashi, The behaviour of a laser-produced bubble near a rigid wall with various configurations.FED vol116 Cavitation' 91, ASME 1991.

5. A.Vogel, W.Lauterborn, R.Timm, Optical and acoustic investigations of the dynamics of laser-produced cavitation bubbles near solid boundary.J.Fluid.Mech.(1989) vol 209 pp299338.

6. B.Ward and D.Emmony, The energies and pressures of acoustic transients associated with optical cavitation in water.Journal of modern optics1990, vol37 $\mathrm{n}^{\circ} 4,803-811$

7. A.P.Alloncle, D.Dufresne, M.Autric; Characterization of pressure waves in liquid using an interferometric method. 19e ISSW Marseille 1993

8. 8.F.Docchio, P.Regondi, M.R.C.Capon, J.Mellerio, "Study of the temporal and spacial dynamics of plasmas induced in liquids by nanosecond Nd:YAG laser pulses. 1: Analysis of the plasma starting times." Applied Optics; vol 27 n $^{\circ} 17$; 1 September 1988; pp 3661-3667 